\title{
Nonlinear parametric model for Granger causality of time series
}

\author{
Daniele Marinazzo, Mario Pellicoro, and Sebastiano Stramaglia \\ TIRES_Center of Innovative Technologies for Signal Detection and Processing, Università di Bari, Bari, Italy; \\ Dipartimento Interateneo di Fisica, Bari, Italy; and Istituto Nazionale di Fisica Nucleare, Sezione di Bari, Bari, Italy
}

(Received 7 February 2006; revised manuscript received 7 April 2006; published 20 June 2006)

\begin{abstract}
The notion of Granger causality between two time series examines if the prediction of one series could be improved by incorporating information of the other. In particular, if the prediction error of the first time series is reduced by including measurements from the second time series, then the second time series is said to have a causal influence on the first one. We propose a radial basis function approach to nonlinear Granger causality. The proposed model is not constrained to be additive in variables from the two time series and can approximate any function of these variables, still being suitable to evaluate causality. Usefulness of this measure of causality is shown in two applications. In the first application, a physiological one, we consider time series of heart rate and blood pressure in congestive heart failure patients and patients affected by sepsis: we find that sepsis patients, unlike congestive heart failure patients, show symmetric causal relationships between the two time series. In the second application, we consider the feedback loop in a model of excitatory and inhibitory neurons: we find that in this system causality measures the combined influence of couplings and membrane time constants.
\end{abstract}

DOI: 10.1103/PhysRevE.73.066216

PACS number(s): 05.45.Tp, 05.10.-a, 87.10.+e, 87.19.La

\section{INTRODUCTION}

Since the seminal paper by Granger [1], detecting causality relationships between two simultaneously recorded signals is one of the most important problems in time series analysis. Applications arise in many fields, like the economy, brain studies, physiology, and many others. The causality between the measured and underground economies in New Zealand has been studied in [2]. Phase synchronization, in electroencephalographic and magnetoencephalographic signals, has been considered in [3], while generalized synchrony in neuronal ensembles has been studied in [4]. Use of nonlinear cellular networks on electroencephalograms from epilepsy patients is proposed in [5], while the experimental detection of weak coupling between self-sustained oscillators has been considered in [6]. The comparison between multivariate and bivariate measures of Granger causality has been presented in [7]; a recent review on applications of multivariate time series analysis to neurophysiological signals is [8]. The analysis of time series from the human cardiorespiratory system is described in [9].

The major approach to causality examines if the prediction of one series could be improved by incorporating information of the other, as proposed by Granger. In particular, if the prediction error of the first time series is reduced by including measurements from the second time series in the regression model, then the second time series is said to have a causal influence on the first time series. As Granger causality was originally developed for linear systems [1], recently some attempts to extend this concept to the nonlinear case have been proposed. In [10] local linear models in reduced neighborhoods are considered and the average causality index, over the whole data set, is proposed as a nonlinear measure. In [11] a radial basis function (RBF) approach has been used to model data, while in [12] a nonparametric test of causality has been proposed, based on the concept of transfer entropy. A recent paper [13] pointed out that not all nonlinear prediction schemes are suitable to evaluate causality between two time series, since they should be invariant if statistically independent variables are added to the set of input variables. This property guarantees that, at least asymptotically, one would be able to recognize variables without causality relationship. The purpose of this work is to use the theoretical results found in [13] to find the largest class of RBF models suitable to evaluate causality, thus extending the results described in [11]. Moreover, we show the application of causality to the analysis of cardiocirculatory interactions and to study the mutual influences in inhibitory and excitatory model neurons.

The paper is organized as follows. In the next section the method is described. In Sec. III the first application, dealing with heart rate and blood pressure in humans, is described, while in Sec. IV we report the analysis of coupled model neurons. Some conclusions are drawn in Sec. V.

\section{METHOD}

Let $\left\{\bar{x}_{i}\right\}_{i=1 \ldots, N}$ and $\left\{\bar{y}_{i}\right\}_{i=1 \ldots \ldots N}$ be two time series of $N$ simultaneously measured quantities. In the following we will assume that the time series are stationary. We aim at quantifying how much $\bar{y}$ is the cause of $\bar{x}$. For $k=1-M$ (where $M=N-m, m$ being the order of the model), we denote $x^{k}=\bar{x}_{k+m}, \mathbf{X}^{k}=\left(\bar{x}_{k+m-1}, \bar{x}_{k+m-2}, \ldots, \bar{x}_{k}\right), \mathbf{Y}^{k}$ $=\left(\bar{y}_{k+m-1}, \bar{y}_{k+m-2}, \ldots, \bar{y}_{k}\right)$, and we treat these quantities as $M$ realizations of the stochastic variables $(x, \mathbf{X}, \mathbf{Y})[14]$. Let us now consider the general nonlinear model

$$
x=w_{0}+\mathbf{w}_{1} \cdot \boldsymbol{\Phi}(\mathbf{X})+\mathbf{w}_{2} \cdot \mathbf{\Psi}(\mathbf{Y})+\mathbf{w}_{3} \cdot \Xi(\mathbf{X}, \mathbf{Y}),
$$

where $w_{0}$ is the bias term, $\{\mathbf{w}\}$ are real vectors of free parameters, $\boldsymbol{\Phi}=\left(\varphi_{1}, \ldots, \varphi_{n_{x}}\right)$ are $n_{x}$ given nonlinear real functions of $m$ variables, $\boldsymbol{\Psi}=\left(\psi_{1}, \ldots, \psi_{n_{y}}\right)$ are $n_{y}$ other real functions of $m$ variables, and $\Xi=\left(\xi_{1}, \ldots, \xi_{n y}\right)$ are $n_{x y}$ functions of $2 m$ variables. The parameters $w_{0}$ and $\{\mathbf{w}\}$ must be fixed to mini- 
mize the prediction error (we assume $M \gg 1+n_{x}+n_{y}+n_{x y}$ ):

$$
\begin{aligned}
\boldsymbol{\epsilon}_{x y}= & \frac{1}{M} \sum_{k=1}^{M}\left[x^{k}-w_{0}-\mathbf{w}_{1} \cdot \boldsymbol{\Phi}\left(\mathbf{X}^{k}\right)-\mathbf{w}_{2} \cdot \boldsymbol{\Psi}\left(\mathbf{Y}^{k}\right)\right. \\
& \left.-\mathbf{w}_{3} \cdot \boldsymbol{\Xi}\left(\mathbf{X}^{k}, \mathbf{Y}^{k}\right)\right]^{2} .
\end{aligned}
$$

We also consider the model

$$
x=v_{0}+\mathbf{v}_{1} \cdot \boldsymbol{\Phi}(\mathbf{X}),
$$

and the corresponding prediction error $\epsilon_{x}$. If the prediction of $\bar{x}$ improves by incorporating the past values of $\left\{\bar{y}_{i}\right\}$, i.e., $\epsilon_{x y}$ is smaller than $\epsilon_{x}$, then $y$ is said to have a causal influence on $x$. We must require that, if $\mathbf{Y}$ is statistically independent of $x$ and $\mathbf{X}$, then $\epsilon_{x y}=\epsilon_{x}$ at least for $M \rightarrow \infty$. This is ensured if, for each $\alpha \in\left\{1, \ldots, n_{x y}\right\}$, an index $i_{\alpha}$ exists such that

$$
\xi_{\alpha}(\mathbf{X}, \mathbf{Y})=\varphi_{i_{\alpha}}(\mathbf{X}) \Gamma_{\alpha}(\mathbf{Y}),
$$

where $\Gamma_{\alpha}$ is an arbitrary function of $\mathbf{Y}$. A complete discussion on this statement may be found in [13]; here we briefly recall the argument that proves it. As $M$ tends to $\infty$, we have

$$
\boldsymbol{\epsilon}_{x y} \rightarrow\left\langle\left[x-w_{0}-\mathbf{w}_{1} \cdot \boldsymbol{\Phi}(\mathbf{X})-\mathbf{w}_{2} \cdot \boldsymbol{\Psi}(\mathbf{Y})-\mathbf{w}_{3} \cdot \boldsymbol{\Xi}(\mathbf{X}, \mathbf{Y})\right]^{2}\right\rangle,
$$

where $\langle\cdot\rangle$ means the expectation over the joint probability distribution of $x, \mathbf{X}$, and $\mathbf{Y}$. If $\mathbf{Y}$ is statistically independent of $x$ and $\mathbf{X}$, one has, for each $\alpha \in\left\{1, \ldots, n_{x y}\right\}$,

$$
\frac{\partial \epsilon_{x y}}{\partial\left(\mathbf{w}_{3}\right)_{\alpha}}=\left\langle\Gamma_{\alpha}(\mathbf{Y})\right\rangle \frac{\partial \epsilon_{x y}}{\partial\left(\mathbf{w}_{1}\right)_{i_{\alpha}}} .
$$

Moreover, for each $\alpha^{\prime} \in\left\{1, \ldots, n_{y}\right\}$,

$$
\frac{\partial \boldsymbol{\epsilon}_{x y}}{\partial\left(\mathbf{w}_{2}\right)_{\alpha}^{\prime}}=\left\langle\psi_{\alpha^{\prime}}(\mathbf{Y})\right\rangle \frac{\partial \boldsymbol{\epsilon}_{x y}}{\partial w_{0}} .
$$

Now, if $\mathbf{w}_{2}=\mathbf{0}$ and $\mathbf{w}_{3}=\mathbf{0}$ then

$$
\frac{\partial \boldsymbol{\epsilon}_{x y}}{\partial\left(\mathbf{w}_{1}\right)_{\alpha^{\prime \prime}}}=\frac{\partial \boldsymbol{\epsilon}_{x}}{\partial\left(\mathbf{v}_{1}\right)_{\alpha^{\prime \prime}}}
$$

for each $\alpha^{\prime \prime} \in\left\{1, \ldots, n_{x}\right\}$, and

$$
\frac{\partial \epsilon_{x y}}{\partial w_{0}}=\frac{\partial \epsilon_{x}}{\partial v_{0}} .
$$

Due to Eqs. (5)-(8), the minimum of $\epsilon_{x y}$ is attained at $\left(w_{0}=v_{0}^{\star}, \mathbf{w}_{1}=\mathbf{v}_{1}^{\star}, \mathbf{w}_{2}=\mathbf{0}, \mathbf{w}_{3}=\mathbf{0}\right)$, where $v_{0}^{\star}$ and $\mathbf{v}_{1}^{\star}$ are the minimizers of $\epsilon_{x}$ : this shows that $\epsilon_{x y}=\epsilon_{x}$ for statistically independent Y. As explained in [13], model (1), with condition (4), is the largest class of nonlinear parametric models suitable to evaluate causality. We remark that $\epsilon_{x y}$ is equal to $\epsilon_{x}$ also at finite $M$, for statistically independent $\mathbf{Y}$, if the probability distribution is replaced by the empirical measure. Exchanging the two time series, one may analogously study the causal influence of $x$ on $y$.

We choose the functions $\boldsymbol{\Phi}, \boldsymbol{\Psi}$, and $\Xi$, in model (1), in the frame of RBF methods, thus generalizing the approach in [11]. We fix $n_{x}=n_{y}=n_{x y}=n \ll M: n$ centers $\left\{\widetilde{\mathbf{X}}^{\rho}, \tilde{\mathbf{Y}}^{\rho}\right\}_{\rho=1}^{n}$, in the space of $(\mathbf{X}, \mathbf{Y})$ vectors, are determined by a clustering procedure applied to data $\left\{\left(\mathbf{X}^{k}, \mathbf{Y}^{k}\right)\right\}_{k=1}^{M}$. To find prototypes we use fuzzy $c$ means [15], a well-known algorithm which in- troduces fuzzy memberships to clusters, so that a point may belong to several clusters with some degree in the range $[0,1]$ : in calculating a cluster center the coordinates of each instance are weighted by the value of the membership function. We then make the following choice for $\rho=1, \ldots, n$ :

$$
\begin{gathered}
\varphi_{\rho}(\mathbf{X})=\exp \left(-\left\|\mathbf{X}-\tilde{\mathbf{X}}^{\rho}\right\|^{2} / 2 \sigma^{2}\right), \\
\psi_{\rho}(\mathbf{Y})=\exp \left(-\left\|\mathbf{Y}-\tilde{\mathbf{Y}}^{\rho}\right\|^{2} / 2 \sigma^{2}\right), \\
\xi_{\rho}(\mathbf{X}, \mathbf{Y})=\varphi_{\rho}(\mathbf{X}) \psi_{\rho}(\mathbf{Y}),
\end{gathered}
$$

$\sigma$ being a fixed parameter, whose order of magnitude is the average spacing between the centers. Condition (4) is satisfied by construction. The RBF model of [11] is recovered by setting $\boldsymbol{\Xi}=\mathbf{0}$ in Eq. (1), i.e., it is constrained to be additive in variables $\mathbf{X}$ and $\mathbf{Y}$; instead the RBF model here proposed can approximate any function of $\mathbf{X}$ and $\mathbf{Y}$. We conclude this section by stressing that, according to our experience, the proposed method is insensitive to details of the clustering procedure used to find prototypes, provided that $n$ is at least two orders of magnitude smaller than $M$.

\section{APPLICATION 1: HEART RATE AND BLOOD PRESSURE}

Now we describe the application to time series of heart rate and blood pressure from patients from an intensive care unit, contained in the MIMIC database [16]. In a healthy subject the heart rate variability (HRV), the pattern of time intervals between heartbeats, and the systolic blood pressure (SBP), the (maximal) pressure within the cardiovascular system as the heart pumps blood into the arteries, are interdependent. Two mechanisms determine the mechanical influence of HRV on SBP. First the Starling law, stating that when the diastolic filling of the heart is increased or decreased by a given volume, the volume of blood which is then ejected from the heart increases or decreases by the same amount: more blood in, more blood out. The second mechanism is diastolic decay, described by the Windkessel model of the capacitative property of arteries [17]. The baroreflex regulation mainly determines the influence of SBP on HRV: it is one of the body's homeostatic mechanisms for maintaining blood pressure. It provides a negative feedback loop in which an elevated blood pressure reflexively causes blood pressure to decrease; similarly, decreased blood pressure depresses the baroreflex, causing blood pressure to rise. This mechanism relies on specialized neurons (baroreceptors) in the aortic arch, carotid sinuses, and elsewhere to monitor changes in blood pressure and relay them to the brainstem. Subsequent changes in blood pressure are mediated by the autonomic nervous system. In healthy subjects the mutual regulation of $\mathrm{HRV}$ and SBP is balanced, whilst it is well known that congestive heart failure (CHF) patients show unbalanced HRV-SBP regulatory mechanism, the feedforward $\mathrm{HRV} \rightarrow \mathrm{SBP}$ coupling being prevalent over baroreflex sensitivity [18].

We consider signals from six CHF patients affected by pulmonary edema, i.e., swelling and/or fluid accumulation in 

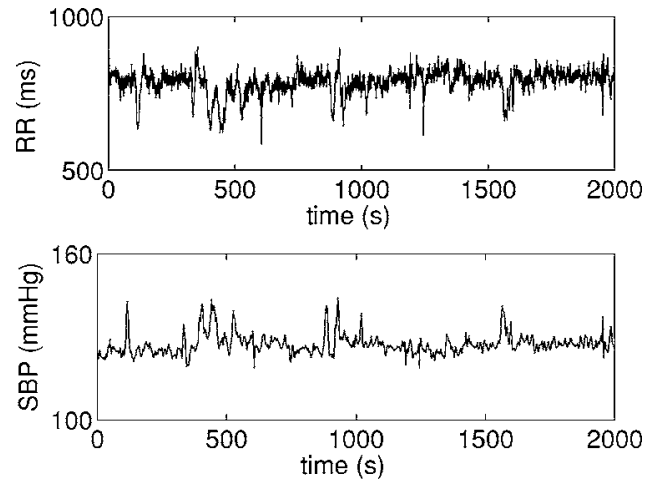

FIG. 1. Time series of heartbeat intervals (above) and systolic blood pressure (below), for a typical patient.

the lungs leading to impaired gas exchange (patients 212, $213,214,225,230$, and 245) and six patients whose primary pathology was sepsis, a condition in which the body is fighting a severe infection and that can lead to shock, a reaction caused by lack of blood flow in the body (patients 222, 224, 269, 291, 410, and 422). HRV and SBP time series are extracted from raw data and resampled at $2 \mathrm{~Hz}$. We denote by $x$ the HRV time series, and by $y$ the SBP time series: Fig. 1 shows the two raw time series for a typical subject. We fix $n=20$ and, at $m=1$, we tune $\sigma$ to minimize the prediction errors $\epsilon_{x}$ and $\epsilon_{y} . \sigma=2.5$ turns out to be nearly optimal for both time series. We then vary $m$ from 1 to 20 , choosing a scaling with the inverse root of $m$, i.e., $\sigma(m)=2.5 / \sqrt{m}$. Figure 2 shows the behavior of $\epsilon_{x}$ and $\epsilon_{y}$ as a function of $m$, for the same subject as in Fig. 1. The optimal value of $m$ corresponds to the knee of the curves, $m=5$; increasing $m$ would increase the complexity of the model without a significant improvement of the fit. In terms of frequency, $m=5$ corresponds to the respiratory band.

In Fig. 3 we depict $\delta_{1}=\left(\epsilon_{x}-\epsilon_{x y}\right) / \epsilon_{x}$ (measuring the influence of SBP on HRV) and $\delta_{2}=\left(\epsilon_{y}-\epsilon_{y x}\right) / \epsilon_{y}$ (measuring the influence of HRV on SBP), as a function of $m$, for CHF and sepsis patients. In the case of sepsis patients, the curves show a symmetric HRV-SBP interdependence, while in CHF patients the $H R V \rightarrow S B P$ influence seems to be dominant, as $\delta_{2}$

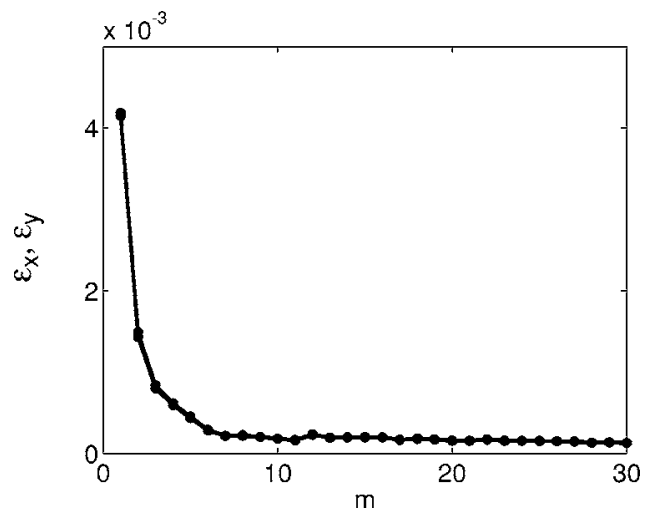

FIG. 2. The prediction errors $\epsilon_{x}$ and $\epsilon_{y}$ (indistinguishable) are plotted vs $m$, the order of the model, for a typical patient.
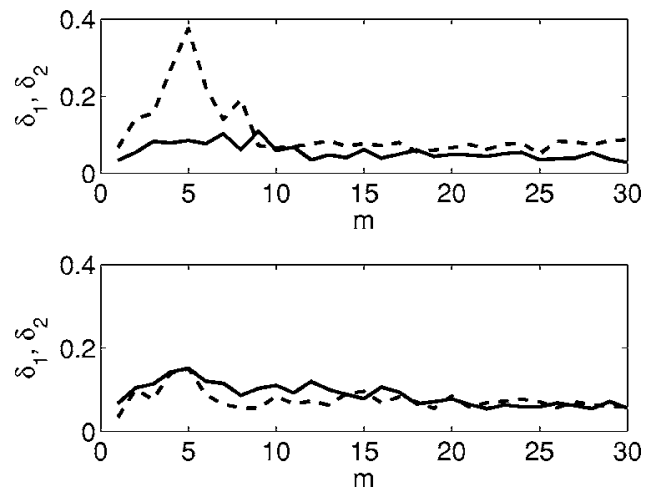

FIG. 3. The directionality indices $\delta_{1}$ (full line, characterizing the influence of systolic blood pressure on heart rate) and $\delta_{2}$ (dashed line, characterizing the influence of heart rate on systolic blood pressure), are plotted vs $m$, averaged over congestive heart failure patients (above), and averaged over sepsis patients (below).

shows a peak at $m=5$. The probability that the $12 \delta_{2}$ values, from all subjects and corresponding to $m=5$, were drawn from the same population has been estimated by Wilcoxon test to be less than $10^{-2}$. The average directionality index $D$ [11], at $m=5$, is equal to 0.82 for CHF patients and to -0.019 for sepsis patients. These results show, on one side, that sepsis condition is not characterized by unbalanced HRV-SBP loop interaction. On the other hand, our findings show that the unbalanced HRV-SBP regulatory mechanism in CHF patients may be also described in terms of Granger causality between HRV and SBP time series. We checked that these results do not depend on our choice of $\sigma(m)$ : varying the dependence of $\sigma$ on $m$ do not change the location of the maximum of the curve $\delta_{2}(m)$ (only the height of the maximum shows a slight variation). Now we compare our results with those from the previous method. In Fig. 4 we depict the quantities $\delta$ as evaluated according to the additive RBF model of [11]: the peak of $\delta_{2}$, for CHF patients, is now almost absent. It is also worth noting that these causal
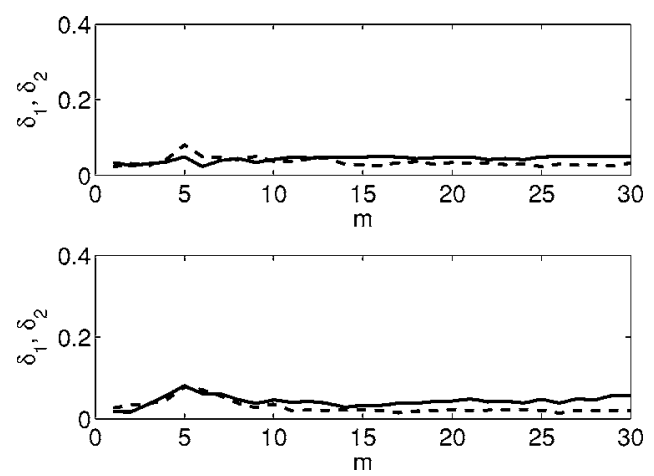

FIG. 4. The directionality indices $\delta_{1}$ (full line, characterizing the influence of systolic blood pressure on heart rate) and $\delta_{2}$ (dashed line, characterizing the influence of heart rate on systolic blood pressure), evaluated by the method proposed in [11], are plotted vs $m$, averaged over congestive heart failure patients (above), and averaged over sepsis patients (below). 

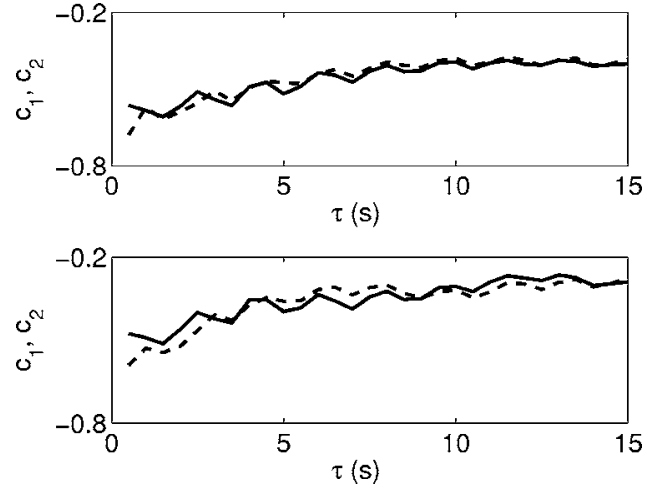

FIG. 5. The cross correlations $c_{1}(\tau)=\langle x(t+\tau) y(t)\rangle-\langle x\rangle\langle y\rangle$ (full line) and $c_{2}(\tau)=\langle x(t) y(t+\tau)\rangle-\langle x\rangle\langle y\rangle$ (dashed line) are plotted vs $\tau$, after averaging over congestive heart failure patients (above), and over sepsis patients (below).

relationships are not evident in terms of cross correlation. The quantities $c_{1}(\tau)=\langle x(t+\tau) y(t)\rangle-\langle x\rangle\langle y\rangle \quad$ and $\quad c_{2}(\tau)$ $=\langle x(t) y(t+\tau)\rangle-\langle x\rangle\langle y\rangle$, depicted in Fig. 5 both for CHF patients and sepsis patients, do not show interesting patterns.

\section{APPLICATION 2: MODEL OF EXCITATORY AND INHIBITORY NEURONS}

As a second application, we consider the interactions in a model of inhibitory and excitatory neurons, which has been studied from different points of view, and with different aims. Many studies have been dedicated to the mechanisms underlying modulation of visual processing by means of attention [19]. Evidence has been reported that in visual area V4 of the brain, the spiking behavior of neurons changes as attention to a particular stimulus is focused inside or outside the neuron's response fields. In particular, increased gammafrequency synchronization and reduced low-frequency synchronization have been shown when the neurons were activated by attended stimuli, and not by distracters. This mechanism of biased attention reveals that some feedback path is present between the retinal neurons and the neurons in deeper layers of visual cortex [20]. In a previous study [21], it has been reported that inhibitory feedback is a way for the neurons to distinguish between spatially correlated and uncorrelated input. So it is important to study causal influences in a closed feedback-feedforward loop, and to understand how the inhibitory feedback, responsible for the selectivity of the attention, modifies the causal relationships between the neurons in the excitatory population. Here we consider the following question: what does causality measure in the case of coupled firing neurons? We show that it measures not merely coupling constants, but the combined influence of couplings and membrane time constants. We consider a model of interacting neurons, two excitatory and one inhibitory, as illustrated in Fig. 6. This is a simplified case of the models in [21-23]. All neurons are leaky integrate-andfire (LIF) neurons with a membrane potential $V$ and an input current $I$ satisfying

$$
\tau_{m} \dot{V}=-V+I
$$

where $\tau_{m}$ is the membrane time constant and the membrane resistance is set to 1 . We denote by $\tau_{E}\left(\tau_{I}\right)$ the membrane

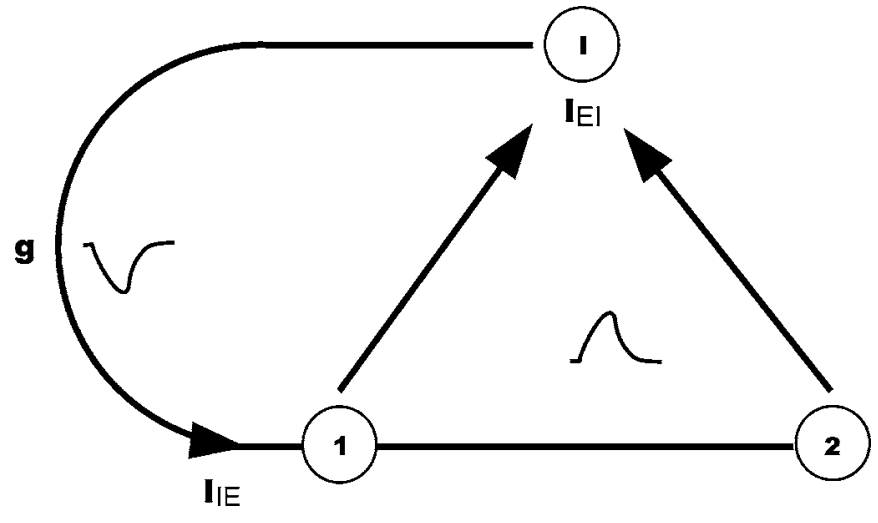

FIG. 6. Neural model architecture.

time constant of excitatory (inhibitory) neurons. Every time the potential of a neuron reaches the threshold value $V_{t h}$, a spike is fired. This resets the potential to the value $V_{R}$; it remains bound to this value for an absolute refractory period $\tau_{R}$. Furthermore we normalize the reset value $V_{R}$ to zero and the threshold value $V_{t h}$ to 1 . The series of spikes $S_{j}^{E}(t)$ $(j=1,2)$ from excitatory neurons provide the input current $I_{I}$ to the inhibitory LIF neuron, given by the convolution of the sum of the spike trains of the excitatory neurons and a standard $\alpha$ function, which mimics an excitatory postsynaptic potential (EPSP). As a consequence of the excitatory input, the inhibitory neuron fires action potentials. As in the case of the excitatory current, the action potential $S^{I}(t)$ of the inhibitory neuron provides the input currents $I_{E, j}$ to the excitatory neurons by convolution of the spike train from the inhibitory neuron and an inhibitory postsynaptic potential (IPSP), given by the same $\alpha$ function used to represent the EPSP, but reversed in sign. This inhibitory feedback is characterized by a gain $g$, and it is delivered after a delay time $\tau_{D}$. Summarizing, for each excitatory neuron $j=1,2$ the total input current $I_{E, j}$ is given by

$$
I_{E, j}(t)=\mu+\eta_{j}(t)-g \int_{0}^{\infty} d \tau S^{I}\left(t-\tau-\tau_{D}\right) \alpha^{2} \tau e^{-\alpha \tau},
$$

while the input current for the inhibitory neuron is

$$
I_{I}(t)=\mu+\int_{0}^{\infty} d \tau\left[S_{1}^{E}(t-\tau)+S_{2}^{E}(t-\tau)\right] \alpha^{2} \tau e^{-\alpha \tau}
$$

where $\mu$ is a constant base current and $\eta_{j}(t)$ represents internal Gaussian white noise with intensity $K$. Note that here we add noise to the excitatory neurons so that they can also fire independently; however, the addition of a small amount of noise also to the input current of the inhibitory neuron would not affect the results. We use $\mu=0.5, K=0.08, \alpha^{-1}=18 \mathrm{~ms}$, and $\tau_{D}=18 \mathrm{~ms}$. $\tau_{R}$ is set to $3 \mathrm{~ms}$, while $\tau_{E}=\tau_{I}=6 \mathrm{~ms}$. Note that the base current is below threshold; therefore the inhibitory neuron only fires in response to excitatory spike input. Moreover, using these parameters, the feedback model is a very stable one, resulting in firing rates from all neurons being almost independent of the values of membrane time 

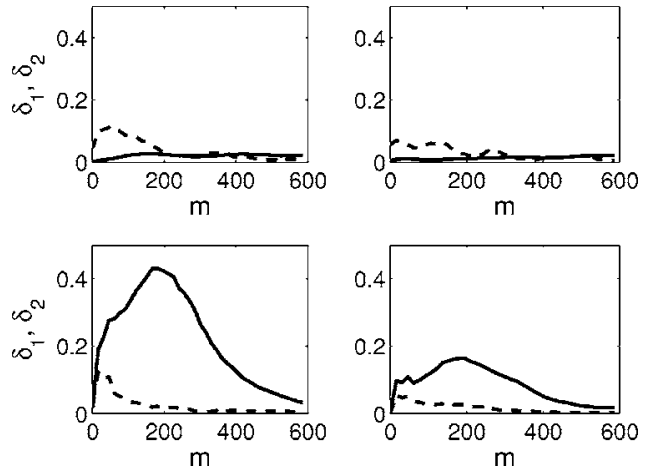

FIG. 7. $\delta_{1}$ (full line, $I \rightarrow E$ ) and $\delta_{2}$ (dashed line, $E \rightarrow I$ ) are plotted vs $m$, for $\tau_{I}=\tau_{E}=6 \mathrm{~ms}$ (left), and $\tau_{I}=18 \mathrm{~ms}, \tau_{E}=6 \mathrm{~ms}$ (right). Above, no feedback. Below, with feedback.

constants and inhibitory feedback gain $g$ used in this study. The firing rates of all the three neurons are always between 40 and $60 \mathrm{~Hz}$. The model equations have been numerically solved by Euler integration using a time step of $0.1 \mathrm{~ms}$. The causal relationships between the time series of membrane potentials from neurons are then evaluated using the proposed RBF approach with $n=20$ and $\sigma=2.5 / \sqrt{m}$, $m \in[1,600]$. In the absence of inhibitory feedback, i.e., $g=0$, there is a dominant causal influence of the excitatory input on the inhibitory one. On the other hand, when the feedback is switched on $(g=1.2)$, the causal influence is reversed (see Fig. 7), and the inhibitory neuron has more causal influence on the excitatory neuron than vice versa. This is due to the fact that each excitatory neuron sees the same input from the inhibitory neuron, and this tends to synchronize the dynamics of the two excitatory neurons.

It is worth stressing, however, that in this context the measure of causality is not simply related to coupling $g$. Indeed, if we consider the same situation, but with a longer membrane time constant of the inhibitory neuron $\tau_{I}$, the influence of the latter on the excitatory ones is notably reduced (see Fig. 7). This can be explained in the following way. When the time constant is short, the inhibitory neuron behaves as a coincidence detector and inhibitory feedback has the effect
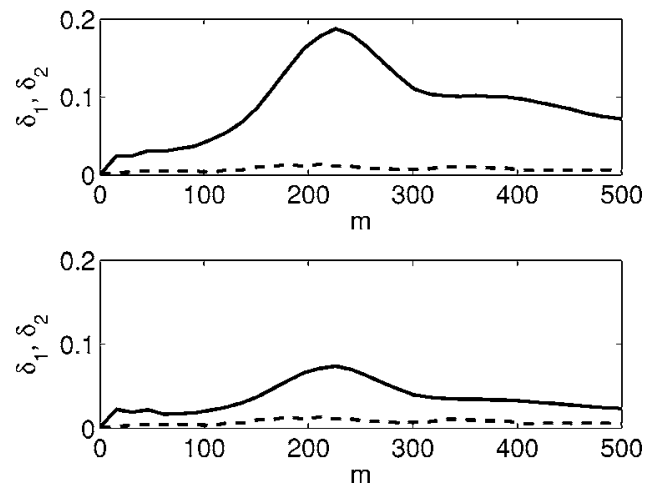

FIG. 8. $\delta_{1}$ (full line, influence of the excitatory neuron without feedback on the one with feedback) and $\delta_{2}$ (dashed line, influence of the excitatory neuron with feedback on the one without feedback) are plotted vs $m$, for $g=1.2 . \tau_{I}=\tau_{E}=6 \mathrm{~ms}$ (top); $\tau_{I}=18 \mathrm{~ms}$, $\tau_{E}=6 \mathrm{~ms}$ (bottom).

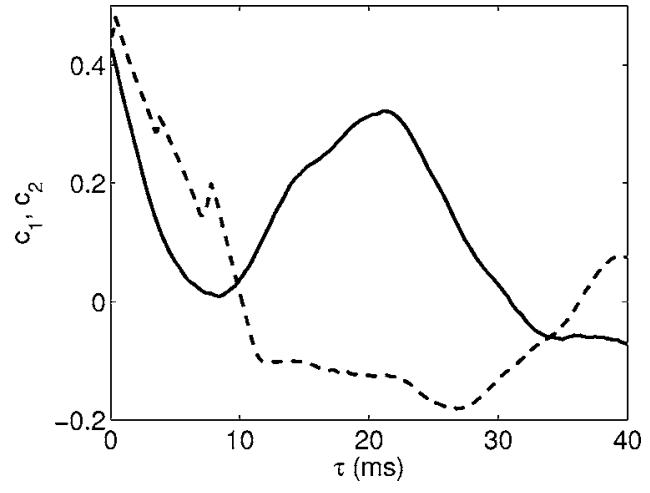

FIG. 9. The cross correlations $c_{1}$ (full line, $I \rightarrow E$ ) and $c_{2}$ (dashed line, $E \rightarrow I$ ) are plotted vs $\tau$, for $g=1.2, \tau_{I}=\tau_{E}=6 \mathrm{~ms}$.

of synchronizing the excitatory neurons [23], which thus tend to fire at the same time. For a longer membrane time constant, the inhibitory neuron acts as an integrator and does not discriminate between two temporally close spikes; the inhibitory neuron then reacts to a smaller number of input spikes, resulting in a decreased causal influence on the two excitatory neurons.

Another interesting situation corresponds to only one, out of the two, excitatory neuron receiving inhibitory feedback: we then investigate the causal relationships between the two excitatory neurons, as mediated by the inhibitory one. In absence of inhibitory feedback there is no causality involved. As the feedback is switched on, as is clear in Fig. 8, a significant causal influence, of the neuron which does not receive the feedback on the other, is observed. Even in this case, the causal influence depends also on the membrane time constant of the inhibitory neuron, which may act as a coincidence detector or as an integrator, thus considering two successive spikes from the two excitatory neurons as two separates spikes, or as one, respectively.

Finally, we report the analysis of this system by standard cross correlations $c_{1}(\tau)$ and $c_{2}(\tau)$ (see the previous section). In Fig. 9 these quantities are plotted, versus $\tau$, for $\tau_{I}=\tau_{E}=6 \mathrm{~ms}$ and $g=1.2 . c_{1}$ shows a peak at $\tau \sim 20 \mathrm{~ms}$ (the same delay at which $\delta_{1}$ shows its peak), while $c_{2}$ is higher than $c_{1}$ for low values of $\tau$.

\section{CONCLUSIONS}

We have generalized a previous RBF approach to Granger causality: the proposed model is not constrained to be additive in variables from the two time series and can approximate any function of these variables, still being suitable to evaluate causality. We have considered here two interesting applications. In the first one, we have studied time series of heart rate and blood pressure in congestive heart failure patients and patients affected by sepsis. Congestive heart failure patients show an unbalanced HRV-SBP regulatory mechanism, while sepsis patients have shown symmetric causal relationships between the two time series. As a second application, we have considered the feedback loop in a model of excitatory and inhibitory neurons and pointed out that in this class of systems Granger causality is not only 
related to couplings, but measures the combined influence of couplings and membrane time constants. The two applications here considered show the usefulness of the proposed approach.

\section{ACKNOWLEDGMENTS}

Valuable discussions with Nicola Ancona and Stan Gielen are warmly acknowledged.
[1] C. W. J. Granger, Econometrica 37, 424 (1969).

[2] D. E. A. Giles, Appl. Econom. Lett. 4, 63 (1997).

[3] P. Tass et al., Phys. Rev. Lett. 81, 3291 (1998); M. Le van Quyen et al., Brain Res. 792, 24 (1998).

[4] S. J. Schiff et al., Phys. Rev. E 54, 6708 (1996); M. Wiesenfeldt, U. Parlitz, and W. Lauterborn, Int. J. Bifurcation Chaos Appl. Sci. Eng. 11, 2217 (2001).

[5] R. Sowa et al., Phys. Rev. E 71, 061926 (2005).

[6] M. G. Rosenblum et al., Phys. Rev. E 64, 045202(R) (2001); M. Palus and A. Stefanovska, ibid. 67, 055201(R) (2003).

[7] K. J. Blinowska, R. Kus, and M. Kaminski, Phys. Rev. E 70, 050902(R) (2004).

[8] E. Pereda, R. Quian Quiroga, and J. Bhattacharya, Prog. Neurobiol. 77, 1 (2005).

[9] M. G. Rosenblum et al., Phys. Rev. E 65, 041909 (2002).

[10] Y. Chen et al., Phys. Lett. A 324, 26 (2004).

[11] N. Ancona, D. Marinazzo, and S. Stramaglia, Phys. Rev. E 70, 056221 (2004).
[12] P. F. Verdes, Phys. Rev. E 72, 026222 (2005).

[13] N. Ancona and S. Stramaglia, Neural Comput. 18, 749 (2006).

[14] Usually both times series are normalized in the preprocessing stage, i.e., they are linearly transformed to have zero mean and unit variance.

[15] J. C. Bezdek, Pattern Recognition with Fuzzy Objective Function Algorithms (Plenum Press, New York, 1981).

[16] http://www.physionet.org/

[17] Mechanisms of Blood Pressure Waves, edited by K. Miyakawa, C. Polosa, and H. P. Koepchen (Springer, Berlin, 1984).

[18] G. D. Pinna et al., J. Am. Coll. Cardiol. 46, 1314 (2005).

[19] J. H. Reynolds et al., J. Neurosci. 19, 1736 (1999).

[20] P. Fries, J. H. Reynolds, A. E. Rorie, and R. Desimone, Science 291, 1560 (2001).

[21] B. Doiron et al., Phys. Rev. Lett. 93, 048101 (2004).

[22] D. Marinazzo, H. J. Kappen, and S. Gielen (unpublished).

[23] C. Börgers and N. Kopell, Neural Comput. 15, 509 (2003). 